\title{
Solitary Fibrous Tumor of the Seminal Vesicle: A Systematic Literature Review and Case Presentation
}

\author{
DANIELE CROCETTI ${ }^{1}$, PAOLO SAPIENZA ${ }^{1}$, ANTONIETTA LAMAZZA $^{1}$, FRANCESCA DE FELICE $^{2}$, \\ GIOIA BRACHINI ${ }^{1}$, ELENA DESIATO ${ }^{1}$, ANDREA MINGOLI $^{1}$, ENRICO FIORI $^{1}$ and ALESSANDRO DE CESARE ${ }^{1}$ \\ ${ }^{1}$ Department of Surgery "Pietro Valdoni", "Sapienza" University of Rome, Rome, Italy; \\ ${ }^{2}$ Department of Radiology, Radiotherapy, Oncology and Pathology, "Sapienza" University of Rome, Rome, Italy
}

\begin{abstract}
Background/Aim: Solitary fibrous tumors (SFTs) are ubiquitous mesenchymal neoplasms that have an unpredictable biological behavior. Histological criteria for this type of malignancy are uncertain. Clinical characteristics, diagnostic and treatment options of SFTs originating in the seminal vesicle are presented in this review article. Materials and Methods: A systematic review including the following databases: Scopus, Embase and Medline from 1960 until the end of March 2021 was performed according to the Preferred Reporting Items for Systematic Reviews (PRISMA) guidelines. Results: We found seven patients affected with SFTs of seminal vesicle, in which we added our own case, making a total of 8 patients. Mean age at presentation was $55 \pm 7$ years. Mean size of the SFTs was $9 \pm 2$ $\mathrm{cm}$ and the right seminal vesicle was preferentially involved. The majority of patients were symptomatic and presenting symptoms were hematuria, dysuria, hematospermia, urinary increased frequency and urgency. Abdominal ultrasonography, computed tomography (CT) scan, and magnetic resonance (MRI) were the diagnostic tools. Trans-rectal ultrasound-guided core biopsy was also used. Seven (87\%) patients had open surgery. Adjuvant radiotherapy after $R 0$ resection was used in 1 patient. Conclusion: The treatment of SFTs located in the seminal vesicle necessitates a radical surgical resection to obtain acceptable results in terms of local recurrence and distant metastases.
\end{abstract}

This article is freely accessible online.

Correspondence to: Daniele Crocetti, MD, Ph.D., Department of Surgery "Pietro Valdoni" "Sapienza" University of Rome, Viale del Policlinico, 155, 00166 Rome, Italy. Tel/Fax: +39 0649972197, email: daniele.crocetti@uniroma1.it

Key Words: Solitary fibrous tumors, hemangiopericytoma, seminal vesicle tumor, review.
Klemperer et al. $(1,2)$ first described, in 1931, a solitary fibrous tumour arising from the visceral pleura; and in the next decades many other sporadic cases of pleural and extrapleural sites have been reported in the literature (3-5). Solitary fibrous tumours (SFTs) are ubiquitous mesenchymal neoplasms arising from CD34-positive dendritic interstitial cells (6) and, in 2013, since they share the same genetic origin with the hemangiopericytomas (HPCs) i.e., NAB2 and STAT6 genes fusion at different exon locations (7), they were unified as a single tumor entity. These tumors are observed in middle-aged adults ranging between 20 and 70 years old with no sex predilection and occasionally occur in childhood and adolescence. SFTs have an unpredictable biological behaviour and histological criteria for malignancy are uncertain; however, the majority of SFTs have a benign clinical course. Characteristics of malignant SFTs are hypercellularity showing $\geq 4$ or more mitotic figures per 10 high power fields (HPF), necrosis or nuclear pleomorphism, and tumour size greater than $5 \mathrm{~cm}$ in diameter (8).

We aimed to discuss the clinical characteristics, the diagnostic and treatment options of SFTs originating in the seminal vesicle. We also performed a complete systematic review of the literature encompassing a period of 60 years (from 1960 to February 2021) with no language restrictions to collect all cases of this rare tumor located in the seminal vesicle.

\section{Materials and Methods}

Literature search. This systematic review was performed following the Preferred Reporting Items for Systematic Reviews (PRISMA) guidelines (9). The literature search was performed on March 15, 2021 included the following database: Scopus, Embase and Medline up to the end of March 2021. Hand searching of reference lists of relevant studies and previous review articles was also performed. The combination of the following words was used: "solitary fibrous tumor" AND "seminal vesicle", "hemangiopericytoma" AND "seminal 
vesicle" and "pelvic solitary fibrous tumor". No language restrictions were applied. Articles were included if they had enough information regarding familial occurrence, symptoms, diagnostic tools, tumours characteristics (size, location, histological features), neo- or adjuvant therapies, surgical treatment, and follow-up (local recurrence or distant metastases). In the case of duplicate publications, the latest and/or the most complete study was included. The Pubmed function "related articles" was used to find further articles.

Data extraction. Two independent reviewers (DC and PS) extracted all available data from each study using a predefined database form. A high inter-observer agreement resulted at the end of analysis. The information included the names of the authors, title of the study, journal in which the study was published, country and year of the study, familial occurrence, symptoms, diagnostic tools, pathologic features, neo- or adjuvant therapies, surgical treatment, and followup (local recurrence or distant metastases). After completing the data extraction from the included papers, the two independent reviewers discussed the results of the collected data and, if discrepancies were present, a consensus was reached by mutual agreement on the accuracy of the data.

Statistical analysis. Data were entered into a computer spreadsheet and statistically analysed with the SPSS 21 software for Mac OSX 10.9.3 (Apple Inc. Cupertino, CA, USA). Data were expressed as mean \pm standard deviation $(\mathrm{SD})$.

\section{Results}

Case report. A 58-year-old white Caucasian man presented at our Institution for an acute onset of lower back pain and lumbosciatalgia. Physical examination revealed a palpable firm mass in the lower abdominal quadrant. Hematochemical tests were within the normal range. An abdominal ultrasound demonstrated a heterogeneous abdominal mass $11 \mathrm{~cm}$ in diameter located in pelvis. Computed tomography (CT scan) revealed a multicystic, well circumscribed tumor measuring $12 \mathrm{~cm}$ in diameter, having a strict contiguity with the prostate (Figure 1). Apparently the tumor had a non-organinfiltrative growth without locoregional lymphadenopathy or distal metastases. For further characterization, the patient also underwent magnetic resonance imaging (MRI), showing a close contact with the posterior bladder wall and the suspicion of an origin from the right seminal vesicle (Figure 2). Surgical excision of the neoplasm was planned. An enbloc mass resection including both vesicle and the prostate was performed. Pathology revealed a high mitotic activity ( $>5$ per HPF), necrosis, high nuclear atypia and high cellularity and, therefore the patient underwent a 3dimensional conformal multiple field technique to a total dose of $50.4 \mathrm{~Gy}$ (1.8 Gy fraction) to the surgical bed, with 6 to $15 \mathrm{MV}$ energy photons. A local recurrence occurred after 22-month. MRI demonstrated the presence of peritoneal carcinosis with a local recurrence of $4 \mathrm{~cm}$ in diameter, without distant metastases. The patient is still alive at 14months after recurrence without other adjuvant therapies.
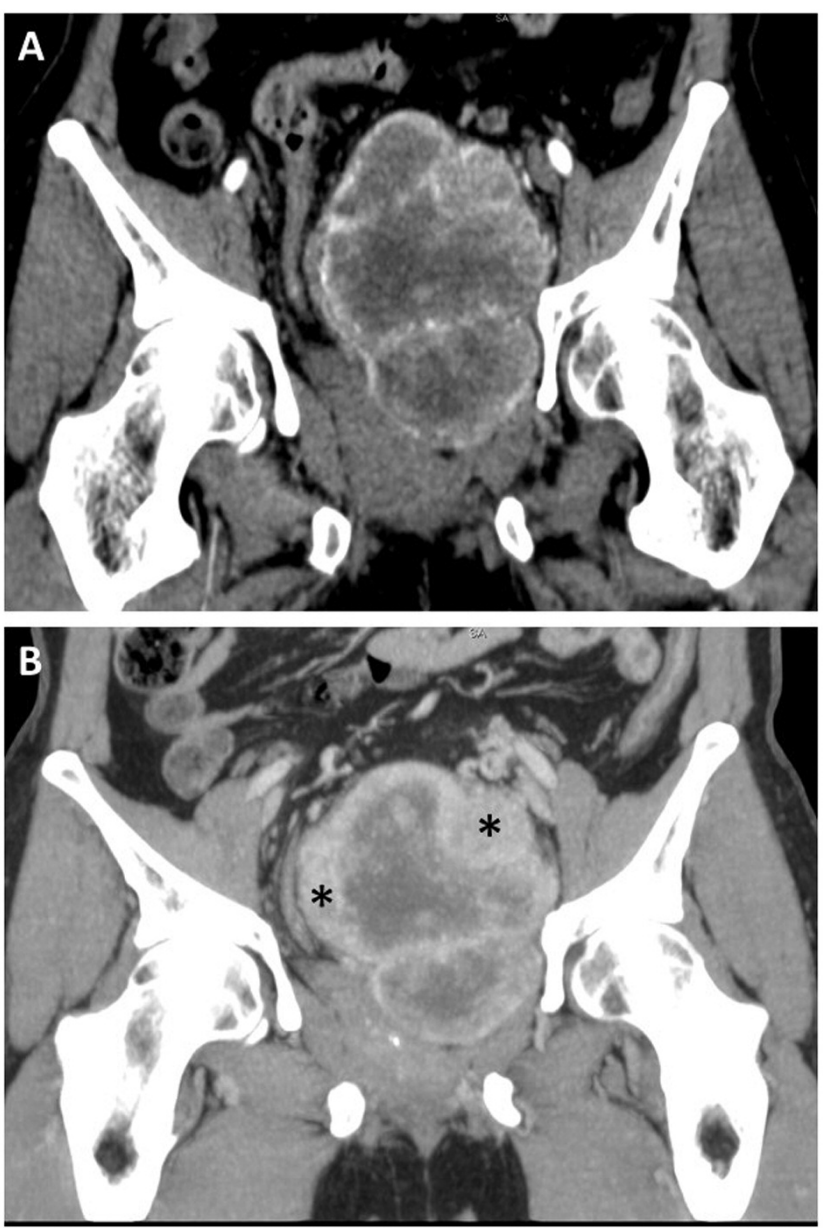

Figure 1. (A) CT scan showing that the solid part of the tumor has an enhancement in the arterial phase. (B) A delayed reinforcement (asterisks) in the venous phase is depicted.

Systematic review analysis. The PRISMA flow chart for systematic review is schematically reported in Figure 3. The initial search produced 17 potentially relevant articles. Following the removal of duplicates and/or not related articles, 6 articles $(2,6,10-13)$ (7 patients) were available and, therefore, were included in our systematic review. Seven patients were found, affected with SFTs of seminal vesicle, in which we added our own case, making a total of 8 patients (Table I). Five articles included a clinical case and 1 article a small series of two cases. All articles were written in English. The studies were performed in Asia [3], north America [2] and Europe ( 2 including the present study), demonstrating no racial or geographical differences.

Demographics. Mean age at presentation was $55 \pm 7$ years (range: min. 46 - max. 66; median 54 years). No specific information regarding past medical history was available but 

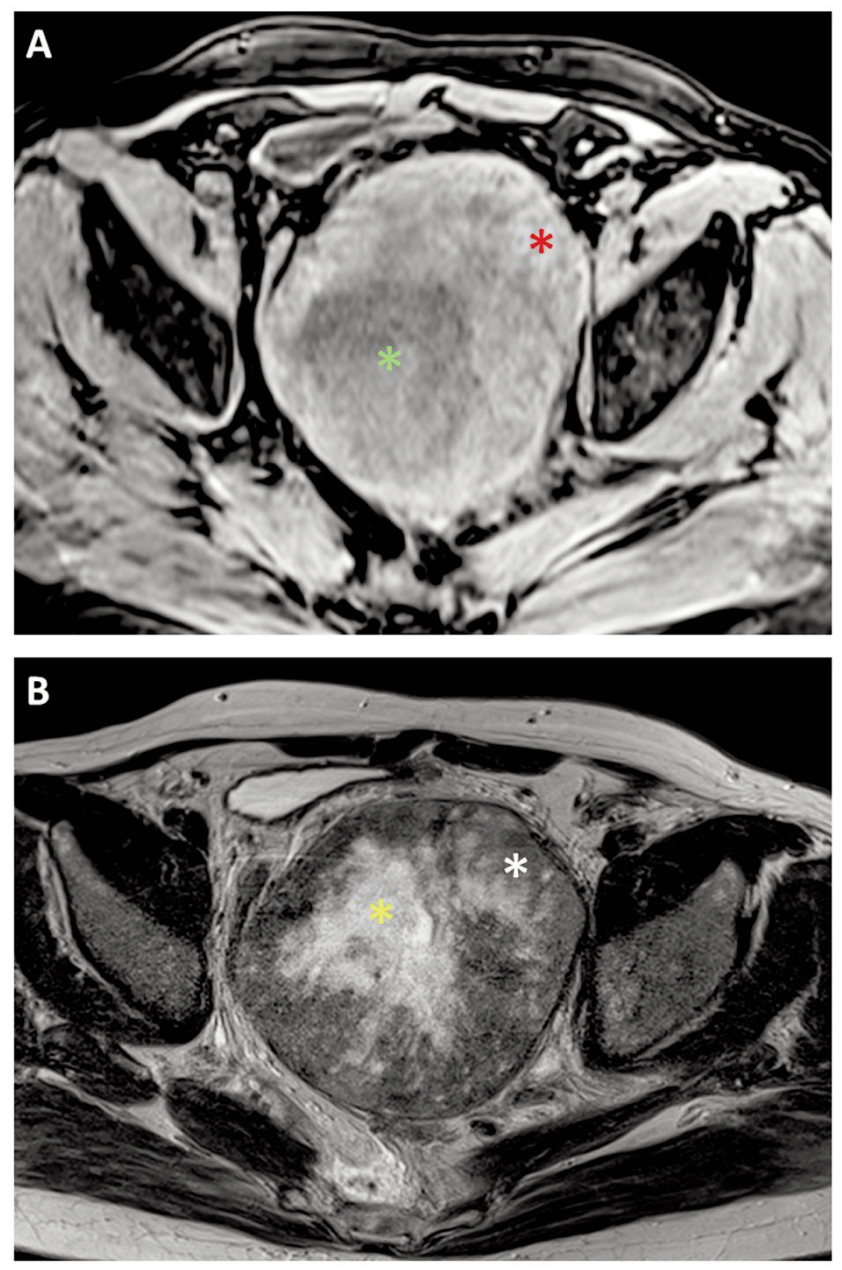

Figure 2. On MRI the solid part of the tumor (A; red asterisk) exhibits a heterogeneous isointensity on T1-weighted imaging and heterogeneous hypointensity on T2-weighted imaging ( $B$; white asteriks) whereas, the cystic part of the tumor a uniform hypointensity on T1weighted imaging (A; green asterisk) and a uniform hyperintensity on T2-weighted imaging ( $B$; yellow asterisk).

one patient accidentally discovered the tumor during hospitalization for acute pancreatitis. No familial predisposition for SFTs location was reported or studied. No patients had synchronous localizations or history of other SFTs location.

Patient clinical presentation. The majority of patients $n=5$ (63\%) was symptomatic whereas $n=3$ (37\%) was asymptomatic. Symptoms, when present, were specific in 3 patients and consisted of haematuria, dysuria, and increased frequency and urgency in $2(25 \%)$, hematospermia in 1 (12\%) and not specific in 2 consisting of left groin discomfort in $1(12 \%)$, and lumbosciatalgia in $1(12 \%)$.

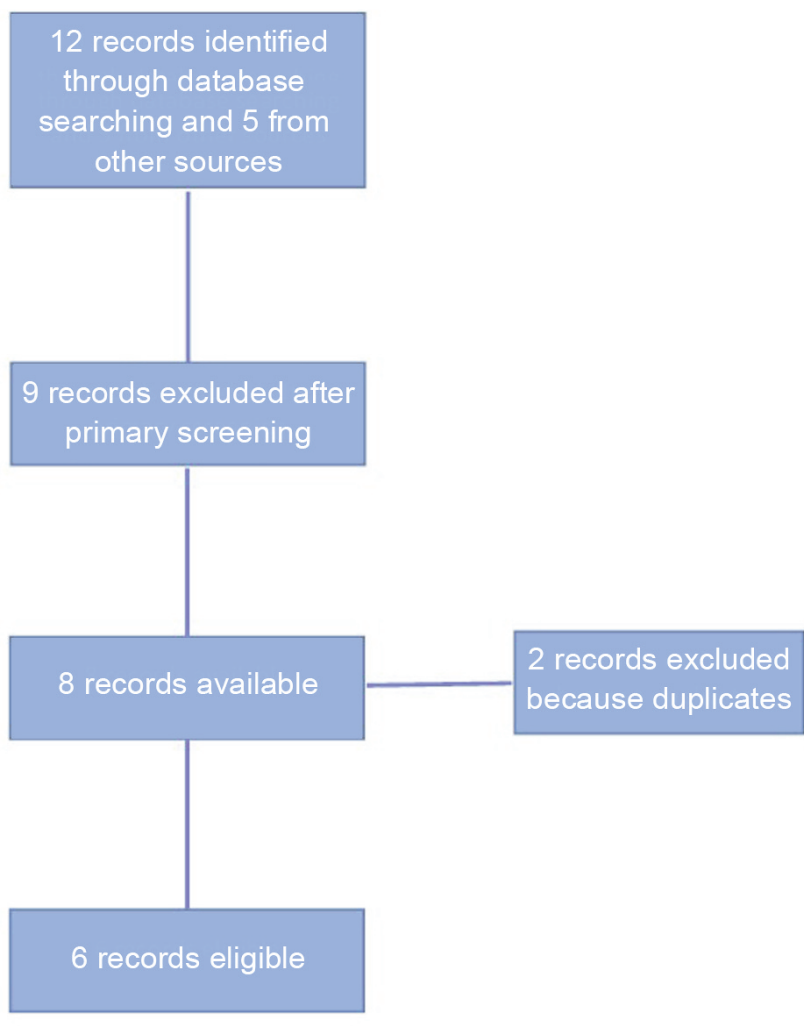

Figure 3. PRISMA flow chart.

Diagnostic tools. Abdominal ultrasonography, computed tomography (CT) scan, and magnetic resonance (MRI) were the diagnostic tools and used alone or in combination to study tumor characteristics. Trans-rectal ultrasound-guided core biopsy was successfully performed in 3 (37\%) patients for pathologal purposes. Abdominal ultrasonography was performed in $3(37 \%)$ patients, but it was not specific in identifying tumor origin, local invasion or eventual distant metastasis. CT scan was performed in $6(75 \%)$ patients demonstrating a well-circumscribed neoplasm; in 2 patients the eventual use of CT scan for preoperative diagnosis is not reported. MRI had a limited role, and it was performed in 4 (50\%) patients. MRI was always able to characterize the muticystic nature of this tumor, but this pathological feature was also noted with the CT scan.

Tumor characteristics. Mean size of the SFTs was $9 \pm 2 \mathrm{~cm}$ (range: $\min .5$ - $\max .12 \mathrm{~cm}$; median $9.5 \mathrm{~cm}$ ). The right seminal vesicle (6 cases, $85 \%$ ) was preferentially involved and in $1(12 \%)$ case the seminal vesicle involved was not specified. A malignant tumor was diagnosed in $4(50 \%)$ patients and the remaining $4(50 \%)$ had a benign tumor. 
in vivo $35: 1945-1950(2021)$

Table I. Demographics, clinical presentation and tumor characteristics of patients included in the literature.

\begin{tabular}{|c|c|c|c|c|c|c|c|c|}
\hline & Age & Symptoms & Side & $\begin{array}{c}\text { Tumor } \\
\text { size }(\mathrm{cm})\end{array}$ & $\begin{array}{l}\text { Histopathological } \\
\text { features }\end{array}$ & $\begin{array}{l}\text { Chemo- } \\
\text { radiotherapy }\end{array}$ & Follow-up & $\begin{array}{l}\text { Local } \\
\text { recurrence }\end{array}$ \\
\hline \multirow{2}{*}{ Westra et al. (10) } & 46 & Hematospermia & RSV & 5 & Malignant & None & 8 & None \\
\hline & 47 & Left groin discomfort & RSV & - & Malignant & None & 15 & None \\
\hline Wiessner et al. (11) & 50 & Asymptomatic & LSV & 10 & Benign & None & 24 & None \\
\hline Wey et al. (13) & 65 & $\begin{array}{l}\text { Hematuria, dysuria, } \\
\text { increased frequency } \\
\text { and urgency }\end{array}$ & RSV & 9 & Benign & None & 14 & None \\
\hline Funahashi et al. (6) & 56 & Asymptomatic & RSV & 9.5 & Benign & None & 8 & None \\
\hline Khandelwal et al. (12) & 52 & $\begin{array}{c}\text { Increased urinary } \\
\text { frequency and urgency }\end{array}$ & RSV & - & Malignant & Yes & 2 & Yes \\
\hline Zhao et al. (2) & 66 & Asymptomatic & - & 12 & Benign & None & 6 & None \\
\hline Crocetti et al. & 58 & Lumbosciatalgia & RSV & 11.5 & Malignant & Yes & 31 & Yes \\
\hline
\end{tabular}

RSV: Right seminal vesicle; LSV: left seminal vesicle.

Surgical procedure. All patients underwent elective surgery. Seven $(88 \%)$ patients had open surgery and 1 (12\%) underwent laparoscopic resection. No intra- or postoperative mortality or major complications were recorded. $\mathrm{R} 0$ resection was obtain in $7(88 \%)$ patients and in $1(12 \%)$ patient, the tumor was not completely resected. In $4(50 \%)$ patients a seminal vesicle tumor removal was sufficient to obtain a R0 resection. In the remaining $4(50 \%)$ cases a multi-organ surgical removal was required but a R0 resection was accomplished in 3. Specifically, a pelvic exenteration with ileal neobladder and ileoileostomy, a cystoprostatectomy with creation of an ileal conduit for urinary diversion, a distal ureterectomy with excision of bladder cuff, and a radical prostatectomy were performed.

Chemo-radiotherapy regimen. One patient underwent adjuvant radiotherapy after $\mathrm{R} 0$ resection, and another patient underwent 60-Gy radiotherapy delivered in 30 fractions for local tumor persistence.

Follow-up. The mean follow-up was $13 \pm 9$ months (range min. 2 - max. 31 months; range 29 months). One patient had a local recurrence at 22 months. The patient, who had an incomplete tumor resection, was successfully reoperated 2 months after radiotherapy.

\section{Discussion}

SFTs represent approximately less than $2 \%$ of all soft tissue tumours and very rarely affect the seminal vesicle and are tumours with an unpredictable behaviour $(2,10)$. Our literature review found only 8 patients, including our own, affected with a seminal vesicle localization of this tumor. Although most cases have a benign course, around $25 \%$ can locally recur or metastasize, showing an unfavourable outcome. Malignant behaviour can be predicted on the basis of tumor diameter greater than $10 \mathrm{~cm}$, high cellularity or cellular pleomorphism, intratumoral necrosis or hemorrhage, cell mitoses more than 4/10 HPF and infiltrative growth behaviour (8).

The clinical presentation might be subtle since they are asymptomatic in $37 \%$ of the cases or hematuria, dysuria, hematospermia, or a palpable pelvic mass might be the presenting symptoms $(63 \%)$, but, in these cases, the tumor have already reached a large size. Theoretically, symptoms from compression of the intra-abdominal organs from tumor growth might be also present but since they are not specific, are not reported in the present literature review. The preoperative diagnosis is difficult because of marginal clinical symptoms, and imaging (CT, MRI) might be not diagnostic. CT scan and MRI show radiological characteristics similar to those of other soft tissue tumours, and there are no pathognomonic findings for SFTs (14). We believe that preoperative trans-rectal ultrasound-guided core biopsy is helpful to obtain a histopathological diagnosis assessing the tumour grade, thus permitting to modify the operative strategy.

We recommend that the therapeutic strategies of these patients should be discussed in a multidisciplinary meeting with experienced specialists in sarcomatous tumours to achieve a correct scheduled treatment. Whenever it is feasible, a 
complete surgical removal of the tumor with negative margins should be the strategy of choice because this is the most important prognostic factor influencing the clinical outcome. An incomplete resection is clearly associate with local recurrence and metastatic disease. The role of a laparoscopic approach $(15,16)$ is questionable; in our analysis it was used in only 1 case. We believe that a laparoscopic tumor resection should be reserved to patients affected with a tumor lesser than $10 \mathrm{~cm}$ in maximum diameter (17-19). In the present literature review, we observed that a reiterative surgery even after radiation therapy should be attempted to obtain a complete tumor resection. Therefore, the behaviour of this tumor is not different from the other more frequent intrabdominal sarcomas (20). Unfortunately, there is no evidence that adjuvant chemoradiotherapy is beneficial for treatment of seminal vesicle SFTs. Neo adjuvant chemoradiation therapies were never used.

Recently, some Authors have investigated the role of systemic therapies and postoperative radiotherapy in advanced SFTs, suggesting that combining radiotherapy to surgery may reduce the risk for local recurrence $(3,21)$. These studies are promising but a greater number of patients is required to find the most efficacious preoperative, intraoperative or postoperative chemo or radiotherapy regimen. In conclusion, at present, the treatment of SFTs located in the seminal vesicle does not differ from the other sarcomas and a radical surgical resection is the mainstay of treatment.

\section{Conflicts of Interest}

The Authors have no conflicts of interest to declare regarding this study.

\section{Authors' Contributions}

Daniele Crocetti (Conception, design and writing the manuscript), Paolo Sapienza (Conception, design and writing the manuscript), Alessandro De Cesare (Conception and design), Antonietta Lamazza (Analysis and interpretation of data), Francesca De Felice (Conception and writing the manuscript), Gioia Brachini (Collection of data), Elena Desiato (Data Analysis), Andrea Mingoli (Conception and design), Enrico Fiori (Conception, design and data analysis).

\section{References}

1 Klemperer P and Coleman BR: Primary neoplasms of the pleura A report of five cases. Am J Ind Med 22(1): 1-31, 1992. PMID: 1415270. DOI: 10.1002/ajim.4700220103

2 Zhao R, Shan Y, Zou L, Zhao H and Zheng S: Solitary fibrous tumor of the seminal vesicle: A case report. Medicine (Baltimore) 98(9): e14660, 2019. PMID: 30817590. DOI: 10.1097/MD.0000000000014660

3 Manica M, Roscigno M, Naspro R, Sodano M, Milesi L, Gianatti A and Da Pozzo LF: Recurrent retroperitoneal solitary fibrous tumor: a case report and review of the literature. Tumori: 300891620974763, 2020. PMID: 33238803. DOI: $10.1177 /$ 0300891620974763
4 Bononi M, De Cesare A, Stella MC, Fiori E, Galati G, Atella F, Angelini M, Cimitan A, Lemos A and Cangemi V: Isolated intestinal neurofibromatosis of colon. Single case report and review of the literature. Dig Liver Dis 32(8): 737-742, 2000. PMID: 11142587. DOI: 10.1016/s1590-8658(00)80340-0

5 De Cesare A, Fiori E, Burza A, Ciardi A, Bononi M, Izzo L, Volpino P, Cavallaro A and Cangemi V: Malignant fibrous histiocytoma of the breast. Report of two cases and review of the literature. Anticancer Res 25(1B): 505-508, 2005. PMID: 15816619.

6 Funahashi Y, Hattori R, Matsukawa Y, Yamamoto T, Mizutani K, Yoshino Y, Komatsu T, Sassa N, Hagikura S and Gotoh M: Solitary fibrous tumor of the seminal vesicle. Aktuelle Urol 41(5): 326-327, 2010. PMID: 20309805. DOI: 10.1055/s-00301247319

7 Louis DN, Perry A, Reifenberger G, von Deimling A, FigarellaBranger D, Cavenee WK, Ohgaki H, Wiestler OD, Kleihues P and Ellison DW: The 2016 World Health Organization classification of tumors of the central nervous system: a summary. Acta Neuropathol 131(6): 803-820, 2016. PMID: 27157931. DOI: $10.1007 / \mathrm{s} 00401-016-1545-1$

8 World Health Organization classification of tumours: Pathology and genetics of tumours of soft tissue and bone. Fletcher, Christopher D.M. (Editor); Unni, Krisjnan (Editor); Mertens, Fredrik (Editor). IARC Press, 2002.

9 Moher D, Liberati A, Tetzlaff J, Altman DG and PRISMA Group: Preferred reporting items for systematic reviews and meta-analyses: the PRISMA statement. J Clin Epidemiol 62(10): 1006-1012, 2009. PMID: 19631508. DOI: 10.1016/j.jclinepi.2009.06.005

10 Westra WH, Grenko RT and Epstein J: Solitary fibrous tumor of the lower urogenital tract: a report of five cases involving the seminal vesicles, urinary bladder, and prostate. Hum Pathol 31(1): 63-68, 2000. PMID: 10665915. DOI: 10.1016/s00468177(00)80200-2

11 Wiessner D, Dittert DD, Manseck A and Wirth MP: Large solitary fibrous tumor of the seminal vesicle. Urology 62(5): 941, 2003. PMID: 14624929. DOI: 10.1016/s0090-4295(03)00695-2

12 Khandelwal A, Virmani V, Amin MS, George U, Khandelwal K and Gorsi U: Radiology-pathology conference: malignant solitary fibrous tumor of the seminal vesicle. Clin Imaging 37(2): 409413, 2013. PMID: 23466005. DOI: 10.1016/j.clinimag. 2012.04.027

13 Wei YC, Li CF, Sung MT, Chen YT, Ko SF, Eng HL and Huang HY: Primary myxoid solitary fibrous tumor involving the seminal vesicle. Pathol Int 56(10): 642-644, 2006. PMID: 16984624. DOI: $10.1111 / \mathrm{j} .1440-1827.2006 .02022 . x$

14 Ros A, Cortés J, Belda T, Magán A, Illán-Gambín FJ, Aracil E and Serra C: Fibrous solitary tumor, a rare and ubiquitous neoplasy. J Surg Case Rep 2020(6): rjaa156, 2020. PMID: 32587681. DOI: $10.1093 /$ jscr/rjaa156

15 Crocetti D, Sapienza P, Pedullà G and De Toma G: Reducing the risk of trocar site hernias. Ann R Coll Surg Eng1 96(7): 558, 2014. PMID: 25245752. DOI: 10.1308/rcsann.2014.96.7.558

16 Cisano C, Sapienza P, Crocetti D and de Toma G: Z-entry technique reduces the risk of trocar-site hernias in obese patients. Ann R Coll Surg Engl 98(5): 340-341, 2016. PMID: 27087329. DOI: $10.1308 /$ rcsann.2016.0114

17 Pedullà G, Sapienza P, Paliotta A, Giordano A, Crocetti D and DE Toma G: Surgical considerations for removal of giant tumor 
of the right adrenal. Anticancer Res 34(9): 5087-5090, 2014. PMID: 25202096.

18 Mingoli A, Sapienza P, Brachini G, Tarantino B and Cirillo B: Surgical treatment of inferior vena cava leiomyosarcoma. J Am Coll Surg 211(1): 145-146, 2010. PMID: 20610268. DOI: 10.1016/j.jamcollsurg.2010.04.001

19 Cavallaro G, Crocetti D, Pedullà G, Giustini S, Letizia C and De Toma G: Retrocaval mass in patient with von Recklinghausen disease: case report. G Chir 33(1-2): 26-30, 2012. PMID: 22357435.

20 Zhang L, Akiyama T, Fukushima T, Iwata S, Tsuda Y, Takeshita K, Kawai A, Tanaka S and Kobayashi H: Prognostic factors and impact of surgery in patients with metastatic soft tissue sarcoma at diagnosis: A population-based cohort study. Jpn J Clin Oncol, 2021. PMID: 33774673. DOI: 10.1093/jjco/hyab033
21 Haas RL, Walraven I, Lecointe-Artzner E, van Houdt WJ, Scholten AN, Strauss D, Schrage Y, Hayes AJ, Raut CP, Fairweather M, Baldini EH, Gronchi A, De Rosa L, Griffin AM, Ferguson PC, Wunder J, van de Sande MAJ, Krol ADG, Skoczylas J, Brandsma D, Doglietto F, Sangalli C and Stacchiotti S: Management of meningeal solitary fibrous tumors/hemangiopericytoma; surgery alone or surgery plus postoperative radiotherapy? Acta Oncol 60(1): 35-41, 2021. PMID: 32988268. DOI: 10.1080/0284186X.2020.1826574

Received April 7, 2021

Revised April 16, 2021

Accepted April 19, 2021 